\title{
3 Research Square \\ CT-based Radiomic Nomogram for Predicting the Severity of Patients With COVID-19
}

\section{Hengfeng Shi}

Anqing Municipal Hospital

\section{Zhihua Xu}

Tongde Hospital Of Zhejiang Province

\section{Guohua Cheng}

Jianpei Technology

Hongli Ji

Jianpei Technology

Linyang $\mathrm{He}$

Jianpei Technology

Juan Zhu

Anqing Municipal Hospital

Hanjin $\mathrm{Hu}$

Anqing Municipal Hospital

\section{Zongyu Xie}

The First Affiliated Hospital of Bengbu Medical College

\section{Weiqun Ao}

Tongde Hospital Of Zhejiang Province

Jian Wang (D119202405@qq.com)

Tongde Hospital Of Zhejiang Province https://orcid.org/0000-0002-8222-9639

\section{Research}

Keywords: COVID-19, X-ray computed tomography, SARS-CoV-2, radiomics

Posted Date: September 14th, 2021

DOI: https://doi.org/10.21203/rs.3.rs-875790/v1

License: (9) (1) This work is licensed under a Creative Commons Attribution 4.0 International License. Read Full License 


\section{Abstract}

Background: The coronavirus disease 2019 (COVID-19) is a pandemic now, and the severe COVID-19 determines the management and treatment, even prognosis. We aim to develop and validate a radiomics nomogram for identifying severe patients with COVID-19. To develop and validate a radiomics nomogram for identifying severe patients with COVID-19.

Methods: There were 156 and 104 patients with COVID-19 enrolled in primary and validation cohorts respectively. Radiomics features were extracted from chest CT images. Least absolute shrinkage and selection operator (LASSO) method was used for feature selection and radiomics signature building. Multivariable logistic regression analysis was used to develop a predictive model, and the radiomics signature, abnormal WBC counts, and comorbidity were incorporated and presented as a radiomics nomogram. The performance of the nomogram was assessed through its calibration, discrimination, and clinical usefulness.

Results: The radiomics signature consisting of 4 selected features was significantly associated with clinical condition of patients with COVID-19 in the primary and validation cohorts $(P<0.001)$. The radiomics nomogram including radiomics signature, comorbidity and abnormal WBC counts, showed good discrimination of severe COVID-19, with an AUC of 0.972, and good calibration in the primary cohort. Application of the nomogram in the validation cohort still gave good discrimination with an AUC of 0.978 and good calibration. Decision curve analysis demonstrated that the radiomics nomogram was clinically useful to identify the severe COVID-19.

Conclusion: We present an easy-to-use radiomics nomogram to identify the severe patients with COVID19 for better guiding a prompt management and treatment.

\section{Background}

The coronavirus disease 2019 (COVID-19), which is caused by severe acute respiratory syndrome coronavirus 2 (SARS-CoV-2), has widely spread all over the world [1-3] due to person to person transmission and it could be described as a pandemic [4]. The epidemic of COVID-19 has attracted worldwide attention and caused a certain degree of social panic.

The incidence and mortality of COVID-19 varied in different countries or territories [1]. According to the previous literature $[5,6], 81 \%$ of the patients with COVID-19 had mild symptoms, but it was the rest of the patients $(19 \%)$ who were in severe and critical conditions that determined the mortality. Because the severe patients with COVID-19 directly influences the clinical management and treatment [7-9], it is crucial for clinicians to evaluate the condition of COVID-19.

Chest computed tomography (CT) can be severed as an important modality to screen, diagnose and evaluate COVID-19 [10-12]. It was reported that the CT features of COVID-19 was manifested as patchy ground-glass opacities (GGOs) with or without consolidation distributed in subpleural areas of bilateral 
lungs [10,13], and increased numbers, greater extent of consolidation on chest CT images were related to progression of COVID-19 $[10,11]$. However, these studies were limited to qualitative analysis, merely focusing on the manifestation of COVID-19 on chest CT images to screen potential new cases of COVID19. The quantitative analysis of correlation between pulmonary abnormalities of COVID-19 on chest CT images and the clinical severity or condition of COVID-19 has not been investigated thoroughly, which may be promising for improving the management of COVID-19.

As the wide application of the artificial intelligence technology for detection of pulmonary nodules has demonstrated great success [14], computer-aided detection and analysis makes quantification and classification of COVID-19 possible. The artificial intelligence evaluation system of COVID-19 was rapidly developed and applied to solve the insufficient expertise of radiologists and speed up screening potential new cases of COVID-19 [14].

Radiomics, as an emerging technique involved with the extraction of high-throughput data from quantitative imaging features and the subsequent association of these parameters with clinical data, has been applied in various diseases. For instance, CT-based radiomics has shown a good performance in predicting of histologic grade, recurrence and metastasis and treatment effect of tumors [15-17]. As far as we know, the existing literature mainly focuses on discriminating COVID-19 and non-COVID-19 pneumonia or other types of viral pneumonia, the predicted results were comparatively ideal $[18,19]$. The average overall case-fatality rate was $2.3 \%$ in confirmed COVID-19 patients, but that was up to $49.0 \%$ in severe patients [20]. Little literature has reported the application of radiomics for evaluation of the severity of COVID-19. If CT-based radiomics can identified the severity of COVID-19, that may guide the clinical treatment and benefited patients. Therefore, the purpose of this study was to apply the artificial intelligence to quantitatively analyze the lung abnormalities associated with COVID-19, and to develop and validate a radiomics nomogram for identifying the severity of COVID-19 who need better management in intensive care units.

\section{Methods}

\section{Patients}

The protocol for this study was approved by the Institutional Review Board of Anqing Municipal Hospital. All patients or their legally authorized representatives were provided written informed consent prior to participation in this study. A total of 260 patients form two hospitals (Anhui, China) with COVID-19 were enrolled from 24 January, 2020 to 1 March, 2020. The patients were grouped into primary cohort (hospital 1 ) and validation cohort (hospital 2) using stratified random resampling method with a ratio of 3:2. The inclusion criteria were: 1)positive for RT-PCR test of SARS-CoV-2; 2) complete clinical data; 3) patients underwent a CT scan. The exclusion criteria were 1) low quality images and 2) normal CT. Their baseline clinical and image data were reviewed retrospectively.

\section{Clinical information}


Basic information including gender, age, comorbidity (hypertension, diabetes mellitus, cardiocerebrovascular disease, the history of surgery for important organs, etc.), laboratory examinations including $C$ reactive protein (CRP), white blood cell (WBC) and lymphocytes were derived from medical records for all patients. A score system based on the number of comorbidities was used to evaluate the state of the patients: none has a score of 0 , one has a score of 1 , two has a score of 2 , more than two has a score of 3.

According to the guideline of American Thoracic Society Criteria [7], the clinical condition of the patients was classified into none-severe (mild and common) and severe (severe and critical) types. Severe patient was defined as: 1) respiratory frequency $\geq 30$ breaths/min; 2) finger oxygen saturation in resting state $\leq$ $93 \%$; 3 ) respiratory distress; 4 ) the presence of shock; 5 ) arteria oxygen tension $(\mathrm{PaO} 2) \leq 300 \mathrm{mmHg} ; 6)$ respiratory failure requires mechanical ventilation; 7 ) patients with organ failure need ICU monitoring and treatment.

\section{CT image acquisition, segmentation, and quantitative analysis}

All patients underwent chest CT scan using a multidetector scanner (16-MDCT, SOMATOM Emotion16, SIEMENS, Germany; 16-MDCT, Definition AS, SIEMENS, Germany; 64-MDCT, Optima CT680, GE, USA) with the following parameters: display field of view (dFOV) $32 \mathrm{~cm}, 300 \mathrm{mAs}$ and $120 \mathrm{Kv}$, slice thickness $5 \mathrm{~mm}$. All CT images were acquired at deep inspiration in the supine position and reconstructed with the slice thickness of $0.625-1.25 \mathrm{~mm}$.

Pleural thickening and pleural effusion were observed in mediastinal window with a window width of 350 Hounsfield unit (HU) and a window level of $40 \mathrm{HU}$.

The flowchart of radiomics procedure is shown in Fig. 1. All CT images of COVID-19 were segmented by a pre-trained Multi-task Unet network. Multi-task Unet is a 2D Unet [21] based network with a single encoder and two parallel decoders, one decoder with attention block to learn the lesion segmentation and another decoder with stacked dilated convolutions to learn the lung segmentation, providing at the same time a more efficient feature encoding and a regularizing effect. At each decoder layer, the features from the corresponding encoder layer are concatenated which help in retaining multi-scale features. Specifically, we concatenated encoder and decoder features, based on the attention block (integration of spatial attention and channel attention) [22] which was learnt for encoder feature and decoder feature separately. By facilitating joint primary of two tasks, not only the model size and inference time were greatly reduced, but also low-level features were effectively reused. The network primary and inference of this experiment were implemented based on Dr. pecker cloud platform (http://www.jianpeicn.com/category/yuepianjiqiren). It is available to public research institutions and is free now around the world for COVID-19 research analysis and prevention.

Primary samples with detailed delineation of each lesion and lung regions were required. Two cardiothoracic radiologists who had 5-15 year's experiences segmented the lesion and lung region using 
ITK-SNAP software (version 3.8.0; http://www.itksnap.org) in lung window with a width of $1500 \mathrm{HU}$ and a level of $-600 \mathrm{HU}$. The margin of the lesion was delineated for each axial slice (Fig. 2.A-C). Then, a 3D regions of interests (ROI) was obtained (Fig. 2.D-F). We split 650 annotated CT scans into 550 for primary and 100 for testing. We tested our model on a holdout $100 \mathrm{CT}$ scans as well to illustrate the robustness of our proposed approach. The average Dice similarity coefficient was 0.973 for the right lung, 0.985 for left lung, and 0.864 for lesion segments.

After segmentation by multi-task Unet, all segmentation results were manually reviewed again in this experiment. Various metrics were computed to quantify the COVID-19 lesion, including volumes of lesion in the whole lung, and volumes of lesion in each lung segment. The GGO and consolidation were distinguished with a threshold value of $-450 \mathrm{HU}$. We used the SimplelTK software tools (http://www.simpleitk.org) to quantify the mean $\mathrm{HU}$ of lung and lesion, number and volume of lesions, volume of GGO and consolidation in double lungs, and the volume of the whole lung automatically. Simultaneously, the ratio of volume of GGO and consolidation in bilateral lungs to total lung volume and to lesion volume in bilateral lungs were calculated respectively. Totally, there were 14 quantitative parameters acquired for feature selection and radiomics model construction.

\section{Statistical Analysis}

R software (version 3.0.1; http://www.Rproject.org) was used for statistical analysis. All the radiological features were normalized between 0 to 1 . The 'caret' package was used to obtain the accuracy, sensitivity and specificity of model. 'pROC' package, 'rms' package and 'rmda' package were used to perform receiver operating characteristic $(\mathrm{ROC})$ analysis, calibration curve analysis and decision curve analysis, respectively. Two-sided $P<0.05$ indicated statistical significance.

\section{Radiological features selection and radiomics signature construction}

The most useful predictive features were selected by using the least absolute shrinkage and selection operator (LASSO) method [23]. Briefly, the optimized hyperparameter $\lambda$ was first determined by using 10fold cross validation with binomial deviance as a criterion. Then the features with non-zero coefficient were selected based on the determined optimal $\lambda$. Finally, LASSO regression was conducted to construct the radiomics signature and a radiomics score (Rad-score) was calculated for each patient via a linear combination of selected and weighted features by their corresponding coefficients.

\section{Individualized prediction model construction}

Besides the radiomics features, the clinical data (termed "clinical feature" later in this article) was also collected. Two clinical features including comorbidity and abnormal WBC counts, which were significantly different between severe and non-severe COVID-19 in univariate regression analysis, were combined with Rad-score to build the nomogram using multivariate logistic regression. The nomogram 
provides the clinicians with a quantitative tool to predict individual probability of severe or none-severe COVID-19.

\section{Performance validation of the nomogram in the primary cohort}

In validation cohort, the same logistic regression formula formed in the primary cohort was used to calculate total points for each patient. Total points were then used as a factor for logistic regression analysis in validation cohort. Finally, two methods including calibration curves analysis and ROC analysis were used to evaluate the performance of nomogram model. Calibration curves were plotted to assess the agreement between the predicted event probability and the observed event probability. The ROC analysis was performed to evaluate the performance of the nomogram. Accuracy, sensitivity and specificity were calculated in both primary cohort and validation cohort.

\section{Clinical Use}

Decision curve analysis was performed to determine the clinical practicability of the nomogram by quantifying the net benefits at different threshold probabilities in both the primary and validation cohorts.

\section{Results}

\section{Clinical characteristics}

There were 156 and 104 patients with COVID-19 enrolled in primary and validation cohorts respectively. Characteristics of patients in the primary and validation cohorts were shown in Table 1. There were significant differences in comorbidity, presence of pleural thickening, CRP increase, abnormal WBC and lymphocytes counts between severe and none-severe patients with COVID-19 in both primary and validation cohorts. Age and presence of pleural effusion differed between severe and none-severe patients with COVID-19 in primary cohort, while they did not differ in validation cohort. A significantly higher proportion of male with severe condition was shown in validation cohort, however, it did not show a significant difference in primary cohort. Note that 9 severe patients were presented with increased WBC counts. 
Table 1

Characteristics of patients in the primary and validation cohorts.

\begin{tabular}{|c|c|c|c|c|c|c|}
\hline \multirow[t]{2}{*}{ Characteristics } & \multicolumn{2}{|l|}{ Primary Cohort } & \multirow{2}{*}{$\begin{array}{l}P \\
\text { value }\end{array}$} & \multicolumn{2}{|c|}{ Validation Cohort } & \multirow{2}{*}{$\begin{array}{l}P \\
\text { value }\end{array}$} \\
\hline & $\begin{array}{l}\text { None-severe } \\
\text { group }(n=130)\end{array}$ & $\begin{array}{l}\text { Severe } \\
\text { group(n = } \\
26)\end{array}$ & & $\begin{array}{l}\text { None-severe } \\
\text { group }(\mathrm{n}=86)\end{array}$ & $\begin{array}{l}\text { Severe } \\
\text { group( } \mathrm{n}= \\
18)\end{array}$ & \\
\hline Male & $68(52.3)$ & $18(69.2)$ & 0.171 & $40(46.5)$ & $14(77.8)$ & 0.031 \\
\hline Age & $42.90(14.71)$ & $\begin{array}{l}58.23 \\
(16.23)\end{array}$ & $\begin{array}{l}< \\
0.001\end{array}$ & 44.58 (14.37) & $\begin{array}{l}49.50 \\
(13.10)\end{array}$ & 0.183 \\
\hline Comorbidity & & & $<.001$ & & & 0.004 \\
\hline 0 & $106(81.5)$ & $11(42.3)$ & & $67(77.9)$ & $11(61.1)$ & \\
\hline 1 & $21(16.2)$ & $6(23.1)$ & & $12(14.0)$ & $5(27.8)$ & \\
\hline 2 & $3(2.3)$ & $5(19.2)$ & & $7(8.1)$ & $0(0.0)$ & \\
\hline 3 & $0(0.0)$ & $4(15.4)$ & & $0(0.0)$ & $2(11.1)$ & \\
\hline $\begin{array}{l}\text { Pleural } \\
\text { thickening }\end{array}$ & & & $\begin{array}{l}< \\
0.001\end{array}$ & & & $\begin{array}{l}<.001 \\
0.001\end{array}$ \\
\hline No & $85(65.4)$ & $4(15.4)$ & & $57(66.3)$ & $1(5.6)$ & \\
\hline Yes & $45(34.6)$ & $22(84.6)$ & & $29(33.7)$ & $17(94.4)$ & \\
\hline $\begin{array}{l}\text { Pleural } \\
\text { effusion }\end{array}$ & & & 0.001 & & & 0.12 \\
\hline No & $124(95.4)$ & $19(73.1)$ & & $80(93.0)$ & $14(77.8)$ & \\
\hline Yes & $6(4.6)$ & $7(26.9)$ & & $6(7.0)$ & $4(22.2)$ & \\
\hline CRP increase & & & 0.004 & & & 0.002 \\
\hline No & $63(48.5)$ & $4(15.4)$ & & $41(47.7)$ & $1(5.6)$ & \\
\hline Yes & $67(51.5)$ & $22(84.6)$ & & $45(52.3)$ & $17(94.4)$ & \\
\hline $\begin{array}{l}\text { WBC } \\
\text { abnormal }\end{array}$ & & & $<.001$ & & & $\begin{array}{l}< \\
0.001\end{array}$ \\
\hline No & $106(81.5)$ & $12(46.2)$ & & $73(84.9)$ & 7 (38.9) & \\
\hline Yes & $24(18.5)$ & $14(53.8)$ & & $13(15.1)$ & $11(61.1)$ & \\
\hline $\begin{array}{l}\text { Lymphocytes } \\
\text { abnormal }\end{array}$ & & & $\begin{array}{l}< \\
0.001\end{array}$ & & & 0.006 \\
\hline No & $76(58.5)$ & $3(11.5)$ & & $48(55.8)$ & $3(16.7)$ & \\
\hline Yes & $54(41.5)$ & 23 (88.5) & & $38(44.2)$ & 15 (83.3) & \\
\hline
\end{tabular}




\begin{tabular}{|c|c|c|c|c|c|c|}
\hline \multirow[t]{2}{*}{ Characteristics } & \multicolumn{2}{|l|}{ Primary Cohort } & \multirow{2}{*}{$\begin{array}{l}P \\
\text { value }\end{array}$} & \multicolumn{2}{|c|}{ Validation Cohort } & \multirow{2}{*}{$\begin{array}{l}\mathrm{P} \\
\text { value }\end{array}$} \\
\hline & $\begin{array}{l}\text { None-severe } \\
\text { group }(n=130)\end{array}$ & $\begin{array}{l}\text { Severe } \\
\text { group }(\mathrm{n}= \\
26)\end{array}$ & & $\begin{array}{l}\text { None-severe } \\
\text { group }(\mathrm{n}=86)\end{array}$ & $\begin{array}{l}\text { Severe } \\
\text { group( } \mathrm{n}= \\
\text { 18) }\end{array}$ & \\
\hline Rad-score & $\begin{array}{l}0.095(0.018 \\
0.222)\end{array}$ & $\begin{array}{l}0.195(0.083, \\
0.337)\end{array}$ & $\begin{array}{l}< \\
0.001\end{array}$ & $\begin{array}{l}0.051(0.012 \\
0.134)\end{array}$ & $\begin{array}{l}0.358(0.207 \\
0.653)\end{array}$ & $\begin{array}{l}< \\
0.001\end{array}$ \\
\hline
\end{tabular}

\section{Feature selection and radiomics signature building}

After analysis of LASSO (Fig. 3.A-B), four factors were selected from quantitative parameters: pleural thickening, total volume of the lesion, ratio of consolidation volume to whole lung volume and ratio of lesion volume to whole lung volume. The selected radiomics features and regression corresponding coefficients were record, then a radiomics score (Rad-score) was calculated and radiomics signature was built. (Table 2)

Table 2

Four selected radiomic features and relevant coefficients in radiomics model

\section{Radiomic features}

pleural thickening

total volume of the lesion

ratio of consolidation volume to whole lung volume

ratio of lesion volume to whole lung volume
Regression coefficient

0.044408621

0.424464103

0.419327051

0.006363664

Note: The radiomics signature was set up as Rad-score $=0.044408621 \times$ pleural thickening + $0.424464103 \times$ total volume of the lesion $+0.419327051 \times$ ratio of consolidation volume to whole lung volume $+0.575642290 \times$ ratio of lesion volume to whole lung volume +0.006363664 .

\section{Diagnostic Validation of Radiomics Signature}

The model score was significantly different between none-severe and severe patients in the primary cohort $(P<0.001)$, which was further confirmed in the validation cohort $(P<0.001)$. The area under the ROC curve (Fig. 3.C-D) for identifying the severe and critical patients based on the model was 0.943 and 0.941 in the primary and validation cohorts, respectively. In primary cohort, the accuracy, sensitivity and specificity for evaluation of the clinical condition were $0.885,0.880$ and 0.885 , respectively.

Correspondingly, the accuracy, sensitivity and specificity were $0.856,0.842$ and 0.859 in validation cohort.

The calibration curve of the radiomics signature for the probability of severe and critical condition of COVID-19 patients indicated good agreement between prediction and observation in the primary cohort (Fig. 3.E), which was then confirmed in the validation cohorts (Fig. 3.F). 
After a logistic regression analysis, the radiomics signature, comorbidity, and abnormal WBC counts were identified as independent predictors of the severity in COVID-19 patients (radiomics signature: OR 95\% C.I., 2.137-5.742; comorbidity: OR 95\% C.I., 1.192-2.087; WBC abnormal: OR 95\% C.I., 8.472-311.790). (Table 3). The model combing the above three independent predictors were developed and presented as the nomogram (Fig. 4).

Table 3

Risk Factors for clinical condition of patients with COVID-19.

\begin{tabular}{|llllll|}
\hline & Coefficient & P value & OR value & \multicolumn{2}{c|}{ OR value (95\% C.I.) } \\
\cline { 5 - 7 } & & & & Lowest & Highest \\
\hline Comorbidity & 1.822 & 0.0014 & 1.577 & 1.192 & 2.087 \\
\hline WBC abnormal & 1.882 & 0.0399 & 51.394 & 8.472 & 311.790 \\
\hline Rad-score & 21.583 & $<0.0001$ & 3.940 & 2.137 & 5.742 \\
\hline Intercept & -8.106 & $<0.0001$ & & & \\
\hline
\end{tabular}

\section{Validation of the Radiomics Nomogram}

The calibration curve of the radiomics nomogram for the probability of severe and critical condition of COVID-19 patients indicated good agreement between prediction and observation models in the primary cohort (Fig. 5.A), which was then confirmed in the validation cohorts (Fig. 5.B). The area under the curve of ROC (Fig. 5.C-D) for identifying the severe and critical patients based on the radiomic nomogram was 0.972 and 0.978 in the primary and validation cohorts, respectively. In primary cohort, the accuracy, sensitivity and specificity for evaluation of the clinical condition were $0.897,0.880$ and 0.900 , respectively. In validation cohort, the corresponding accuracy, sensitivity and specificity were $0.923,0.894$ and 0.929 .

\section{Clinical Use}

The decision curve analysis for the radiomics nomogram was shown in Fig. 7. The decision curve showed that if the threshold probability of a patient is more than $3 \%$, using the radiomics nomogram to identify severe patients adds more benefit than either treat all as severe patients or none severe patients. When radiomics signature was combined with clinical risk factors (radiomics nomogram), an improved benefit net was achieved.

\section{Discussion}

In this study, we developed and validated a radiomics nomogram based on the quantitation of lung abnormalities on CT images caused by COVID-19 to identify the severe patients for guiding a prompt management and treatment. The radiomics nomogram incorporates three items of the radiomics signature, comorbidity and abnormal WBC counts. The radiomics signature successfully stratified 
patients according to their clinical conditions (severe or none-severe). The use of multi-task Unet network, which could segment the lesion or lung abnormalities related to COVID-19 automatically, increased the potential value of the radiomics nomogram in evaluating the clinical condition of patients with COVID-19.

Previous studies $[18,19,24]$ have demonstrated that CT-based radiomics as a superior tool for screening potential new cases of COVID-19, and had a good prediction on discriminating COVID-19 and non-COVID19 pneumonia or other types of viral pneumonia. Mei et al. [24] used CT-based radiomics achieved an AUC of 0.92 and had equal sensitivity as compared to a senior radiologist when applied to a group of 279 cases, CT-based radiomics can be severed as a rapid method for screening COVID-19 patients. Huang et al. [19] summarized 154 patients with viral pneumonia (including 65 cases of influenza pneumonia and 89 cases of COVID-19) to develop a CT-based radiomics model, the results showed radiomics model had a satisfactory performance in distinguishing influenza pneumonia and COVID-19. Nevertheless, it is of great necessity to assess the severity of patients with COVID-19 before treatment, which may greatly determine the clinical prognosis. We firstly assessed the lung abnormalities associated with COVID-19 by quantitative analysis, and then developed and validated a radiomics signature to identify severe COVID19 patients. The results in present study uncovered that the radiomics signature could get a better performance in discriminating the severity of COVID-19 patients with an AUC of 0.943 in primary cohort, which was then further confirmed in validation cohort with an AUC of 0.941. Thus, the radiomics signature was effective for identification of none-severe and severe type COVID-19 patients. Notably, when combined with clinical risk factors including comorbidity and abnormal WBC counts, the discrimination potency was improved with an AUC of 0.972 and 0.978 in the primary and validation cohorts, respectively. Thus, we think that the noninvasive radiomics signature, which makes the most of the chest CT images, may serve as a practical method for identification of none-severe and severe type COVID-19 patients.

The radiomics signature includes four parameters of pleural thickening, total volume of the lesion, ratio of consolidation volume to whole lung volume and ratio of lesion volume to whole lung volume, which were obtained automatically by computer-aided system or Multi-task Unet network. Presently, COVID-19 has reached the stage of a pandemic, which contributed to an extreme shortage of clinicians and radiologists. The application of artificial intelligence technology or computer-aided system, a noninvasive, fast, reproducible technique, to assess the COVID-19 could alleviate the insufficiency of radiologists to some extent. Furthermore, patients with COVID-19 would benefit from a timely and accurate assessment of the severity through radiomics signature before getting a prompt and proper treatment.

It is unexpected that increased total volume of the lesion, ratio of consolidation volume to whole lung volume and ratio of lesion volume to whole lung volume, are associated with severe COVID-19 patients. The more extensive involvement of lung parenchymal, the more severe condition it would be. The appearance of GGO indicates that alveolar cavity is partially filled by fluid and cells to the layer against the alveolar walls[25], while the consolidation sign demonstrates that the disease progresses due to further accumulation of exudates in alveolar cavity and aggravation of interstitial edema [25]. The chest CT features of COVID-19 are manifested as multiple patchy GGOs with or without consolidation 
distributed in subpleural areas of bilateral lungs [15]. When the volume of consolidation increases, more alveolar cavities are filled completely with exudates, resulting in dysfunction of oxygen exchange and oxygenation. Then, a respiratory failure occurs, which is presented as a severe condition. Above all, our study quantified the lesion of GGO and consolidation to investigate its value in identification of severe patients with COVID-19, and to build a useful radiomics signature for clinicians.

Additionally, clinical features including comorbidity and abnormal WBC counts were independent risk factors contributing to worse clinical condition of patients with COVID-19. According to a previous study, presence of comorbidity is an essential factor in determining the prognosis of several diseases, especially pneumonia [26]. Therefore, we also has taken comorbidity into consideration in the present study and found a positive correlation with the severity of COVID-19, which was consistent with the previously study [27]. CRP is an important inflammatory index. Although a significant difference in CRP increase was indicated by univariate analysis in primary and validation cohorts, it was not an independent predictor for identification of clinical condition of COVID-19 in this study. The main reasons may be that 1) CRP is a common signal for responding to inflammation; 2) the change of CRP is analyzed as a categorical variable, which may lead a bias to subtle difference. Moreover, Viral infections in the human body primarily involve damage to the immune system, which presents as decrease in the absolute number of lymphocytes and leukocyte [28]. In this study, we found that leukocyte and lymphocytes differed between severe and none severe patients with COVID-19, which is consistent with the study of Wang D et al [27]. In addition, WBC (leukocyte) is an independent predictor for identification of clinical condition of COVID-19. Interestingly, 9 severe patients presented with an increased WBC counts, which may be ascribed to other infections, such as bacterium. Comprehensively, a severe and critical patient with COVID-19 may be caused by cytokine storm, comorbidity with various infections (9 patients with increased WBC counts) and immune dysfunction. In a word, incorporating clinical features into radiomics nomogram could improve its diagnostic value of none-severe and severe cases with COVID-19.

The most important application of the radiomics nomogram is to guide management and treatment of patients with COVID-19, especially for severe type cases who need additional treatment or care. According to recent reports and recommendations, severe patients with COVID-19 need hospitalized therapy. Besides antiviral therapy, some additional treatment should be added for severe patients [27, 29]. To block cytokine storm, a blood-purifying therapy including plasmapheresis, hemoperfusion is recommended, which can reduce the damage of inflammatory reaction to the body or lung [7]. If possible, convalescent plasma therapy could be a preferred scheme for treatment of severe patients [7]. Using the nomogram, we can quickly and precisely identify the none-severe and severe patients with COVID-19, and prompt a timely additional treatment and care to improve prognosis. On the other hand, COVID-19 is a dynamic disease [14,30], a quantitative radiomics nomogram is helpful to follow up the changes of patients after treatment. To justify the clinical practicability of radiomics nomogram, decision curve analysis was applied in this study. This novel method offers an insight into clinical consequences based on threshold probability, from which the net benefit could be derived (Net benefit is defined as the proportion of true positives minus the proportion of false positives, weighted by the relative harm of falsepositive and false-negative results). The decision curve in our study showed that if the threshold 
probability of a patient was more than $3 \%$, using the radiomics nomogram to identify none-severe or severe patients added more benefit than either treat all as severe patients or none-severe patients.

Admittedly, our study has several limitations. The sample size in our cohort is relatively small. The relationship of radiomics to prognosis has not been studied due to time limitation. Thus, a further study with more cases and prolonged period should be conducted in the future.

\section{Conclusion}

In conclusion, we present an easy-to-use radiomics nomogram to identify the severe patients of COVID-19 for guiding a prompt management and treatment. We believe that both clinicians and COVID-19 patients could greatly profit from the use of the radiomics nomogram.

\section{Abbreviations}

COVID-19: coronavirus disease 2019; CRP: C-reactive protein; CT: computed tomography; dFOV: display field of view ; GGO: Ground ground-glass opacity; HU:Hounsfield unit; LASSO: Least absolute shrinkage and selection operator; RT-PCR: real-time reverse transcriptase polymerase chain reaction; WBC: white blood cell.

\section{Declarations}

\section{Ethics approval and consent to participate}

The protocol for this study was approved by the Institutional Review Board of Anqing Municipal Hospital. All patients or their legally authorized representatives were provided written informed consent prior to participation in this study.

\section{Consent for publication}

Not applicable.

\section{Availability of data and materials}

All data generated or analyzed during this study are included in this published article.

\section{Competing Interests}

The authors declared that there is no conflict of interest.

\section{Authors' contributions}


HS,ZX, GC, HJ, LH, QH, HH, JZ, WA and JW conceived and designed the study. XZ, HH,JZ,HS, ZX,WA,JW and $L H$ contributed to the literature search. ZX, JW, WA and HS contributed to data collection. GC, HS,QH, $\mathrm{HJ}, \mathrm{LH}, \mathrm{JZ}, \mathrm{HH}, \mathrm{JW}, \mathrm{ZX}$ and ZX contributed to data analysis. HS, ZX, WA and JW contributed to data interpretation. LH, QH, HJ, GC, JZ, ZX,WA,HS and JW contributed to the figures. HS,JW, ZX and WA contributed to writing of the report. All authors have read and approved the manuscript.

\section{Funding}

This work was supported by the Coronavirus disease 2019 (COVID-19) emergency plan project of Anqing (2020Z1003).

\section{Acknowledgements}

Not applicable.

\section{References}

1. Lai CC, Wang CY, Wang YH, Hsueh SC, Ko WC, Hsueh PR. Global epidemiology of coronavirus disease 2019 (COVID-19): disease incidence, daily cumulative index, mortality, and their association with country healthcare resources and economic status. Int J Antimicrob Agents. 2020;55(4):105946. doi: 10.1016/j.jijantimicag.2020.105946.

2. Velavan TP, Meyer CG. The COVID-19 epidemic. Trop Med Int Health. 2020;25(3):278-280. doi: 10.1111/tmi.13383.

3. Yang J, Liao X, Yin W, Wang B, Yue J, Bai L, et al. Elevated cardiac biomarkers may be effective prognostic predictors for patients with COVID-19: A multicenter, observational study[J]. The American Journal of Emergency Medicine, 2021, 39:34-41. https://doi.org/10.1016/j.ajem.2020.10.013.

4. Chan JF, Yuan S, Kok KH, To KK, Chu H, Yang J, et al. A familial cluster of pneumonia associated with the 2019 novel coronavirus indicating person-to-person transmission: a study of a family cluster. Lancet. 2020;395(10223):514-523. doi: 10.1016/S0140-6736(20)30154-9.

5. Wang J, Zhu X, Xu Z, Yang G, Mao G, Jia Y, et al. Clinical and CT findings of COVID-19: differences among three age groups. BMC Infect Dis. 2020;20(1):434. doi: 10.1186/s12879-020-05154-9. .

6. Wang C, Pan R, Wan X, Tan Y, Xu L, Ho CS. Immediate Psychological Responses and Associated Factors during the Initial Stage of the 2019 Coronavirus Disease (COVID-19) Epidemic among the General Population in China. Int J Environ Res Public Health. 2020;17(5):1729. doi: 10.3390/ijerph17051729.

7. Metlay JP, Waterer GW, Long AC, Anzueto A, Brozek J, Crothers K, et al. Diagnosis and Treatment of Adults with Community-acquired Pneumonia. An Official Clinical Practice Guideline of the American Thoracic Society and Infectious Diseases Society of America. Am J Respir Crit Care Med. 2019;200(7):e45-e67. doi: 10.1164/rccm.201908-1581ST. 
8. Li T. Diagnosis and clinical management of severe acute respiratory syndrome Coronavirus 2 (SARSCoV-2) infection: an operational recommendation of Peking Union Medical College Hospital (V2.0). Emerg Microbes Infect. 2020 Mar 14;9(1):582-585. doi: 10.1080/22221751.2020.1735265.

9. Wang J, Xu Z, Wang J, Feng R, An Y, Ao W, et al. CT characteristics of patients infected with 2019 novel coronavirus: association with clinical type. Clin Radiol. 2020 Jun;75(6):408-414. doi: 10.1016/j.crad.2020.04.001.

10. Li Y, Xia L. Coronavirus Disease 2019 (COVID-19): Role of Chest CT in Diagnosis and Management. AJR Am J Roentgenol. 2020;214(6):1280-1286. doi: 10.2214/AJR.20.22954.

11. Zhao W, Zhong Z, Xie X, Yu Q, Liu J. Relation Between Chest CT Findings and Clinical Conditions of Coronavirus Disease (COVID-19) Pneumonia: A Multicenter Study. AJR Am J Roentgenol. 2020;214(5):1072-1077. doi: 10.2214/AJR.20.22976.

12. Song F, Shi N, Shan F, Zhang Z, Shen J, Lu H, et al. Emerging 2019 Novel Coronavirus (2019-nCoV) Pneumonia. Radiology. 2020 Apr;295(1):210-217. doi: 10.1148/radiol.2020200274.

13. Lei J, Li J, Li X, Qi X. CT Imaging of the 2019 Novel Coronavirus (2019-nCoV) Pneumonia. Radiology. 2020;295(1):18. doi: 10.1148/radiol.2020200236.

14. Lee SM, Seo JB, Yun J, Cho YH, Vogel-Claussen J, Schiebler ML, et al. Deep Learning Applications in Chest Radiography and Computed Tomography: Current State of the Art. J Thorac Imaging. 2019;34(2):75-85. doi: 10.1097/RTI.0000000000000387.

15. Yi X, Xiao Q, Zeng F, Yin H, Li Z, Qian C, et al. Computed Tomography Radiomics for Predicting Pathological Grade of Renal Cell Carcinoma. Front Oncol. 2021;10:570396. doi:10.3389/fonc.2020.570396

16. Ao W, Cheng G, Lin B, Yang R, Liu X, Zhou S, et al. A novel CT-based radiomic nomogram for predicting the recurrence and metastasis of gastric stromal tumors. Am J Cancer Res. 2021;11(6):3123-3134.

17. Liu Z, Meng X, Zhang $H$, et al. Predicting distant metastasis and chemotherapy benefit in locally advanced rectal cancer. Nat Commun. 2020;11(1):4308. doi:10.1038/s41467-020-18162-9

18. Li Z, Zhong Z, Li Y, Zhang T, Gao L, Jin D, et al. From community-acquired pneumonia to COVID-19: a deep learning-based method for quantitative analysis of COVID-19 on thick-section CT scans. Eur Radiol. 2020;30(12):6828-6837. doi: 10.1007/s00330-020-07042-x.

19. Huang Y, Zhang Z, Liu S, Li X, Yang Y, Ma J, et al. CT-based radiomics combined with signs: a valuable tool to help radiologist discriminate COVID-19 and influenza pneumonia. BMC Med Imaging. 2021;21(1):31. https://doi.org/10.1186/s12880-021-00564-w.

20. Wu ZY, McGoogan JM. Characteristics of and important lessons from the coronavirus disease 2019 (COVID-19) outbreak in China summary of a report of 72314 cases from the Chinese Center for Disease Control and Prevention. JAMA. 2020;323(13):1239-42

21. LaLonde R, Xu Z, Irmakci I, Jain S, Bagci U. Capsules for biomedical image segmentation. Med Image Anal. 2021;68:101889. doi: 10.1016/j.media.2020.101889. 
22. Li C, Ren L. Recent progress on the diagnosis of 2019 Novel Coronavirus. Transbound Emerg Dis. 2020 Jul;67(4):1485-1491. doi: 10.1111/tbed.13620. Epub 2020 May 31. Erratum in: Transbound Emerg Dis. 2020;67(6):3076.

23. Sauerbrei W, Royston P, Binder H. Selection of important variables and determination of functional form for continuous predictors in multivariable model building. Stat Med. 2007;26(30):5512-28. doi: 10.1002/sim.3148.

24. Chen Z, Li X, Li J, Zhang S, Zhou P, Yu X, et al. A COVID-19 risk score combining chest CT radiomics and clinical characteristics to differentiate COVID-19 pneumonia from other viral pneumonias. Aging (Albany NY). 2021;13(7):9186-9224. doi: 10.18632/aging.202735.

25. Jin YH, Cai L, Cheng ZS, Cheng H, Deng T, Fan YP, et al. Evidence-Based Medicine Chapter of China International Exchange and Promotive Association for Medical and Health Care (CPAM). A rapid advice guideline for the diagnosis and treatment of 2019 novel coronavirus (2019-nCoV) infected pneumonia (standard version). Mil Med Res. 2020;7(1):4. doi: 10.1186/s40779-020-0233-6.

26. Ladha KS, Zhao K, Quraishi SA, Kurth T, Eikermann M, Kaafarani HM, et al. The Deyo-Charlson and Elixhauser-van Walraven Comorbidity Indices as predictors of mortality in critically ill patients. BMJ Open. 2015;5(9):e008990. doi: 10.1136/bmjopen-2015-008990.

27. Wang D, Hu B, Hu C, Hu C, Zhu F, Liu X, et al. Clinical Characteristics of 138 Hospitalized Patients With 2019 Novel Coronavirus-Infected Pneumonia in Wuhan, China. JAMA. 2020;323(11):1061-1069. doi: 10.1001/jama.2020.1585.

28. Wu CT, Hsia SH, Huang JL. Influenza B-associated rhabdomyolysis in Taiwanese children. Acta Paediatr. 2010;99(11):1701-4. doi: 10.1111/j.1651-2227.2009.01595.x.

29. Liu W, Tao ZW, Wang L, Yuan ML, Liu K, Zhou L, et al. Analysis of factors associated with disease outcomes in hospitalized patients with 2019 novel coronavirus disease. Chin Med J (Engl). 2020;133(9):1032-1038. doi: 10.1097/CM9.0000000000000775.

30. Chen $\mathrm{H}$, Zeng $M$, Wang $X$, Su L, Xia Y, Yang Q, et al. A CT-based radiomics nomogram for predicting prognosis of coronavirus disease 2019 (COVID-19) radiomics nomogram predicting COVID-19. Br J Radiol. 2021;94(1117):20200634. doi: 10.1259/bjr.20200634.

\section{Figures}




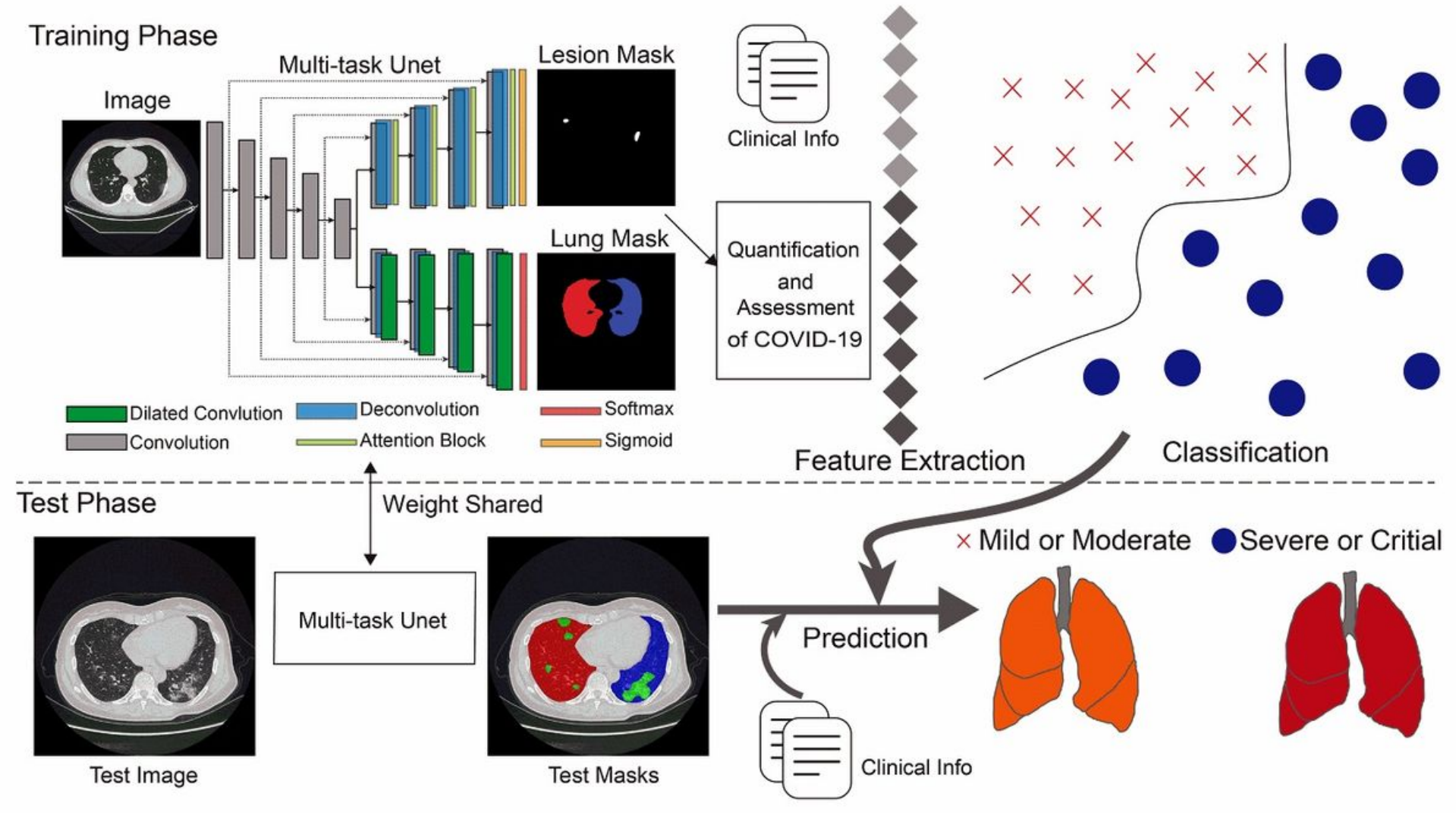

Figure 1

Flowchart of radiomics procedure in this study. 


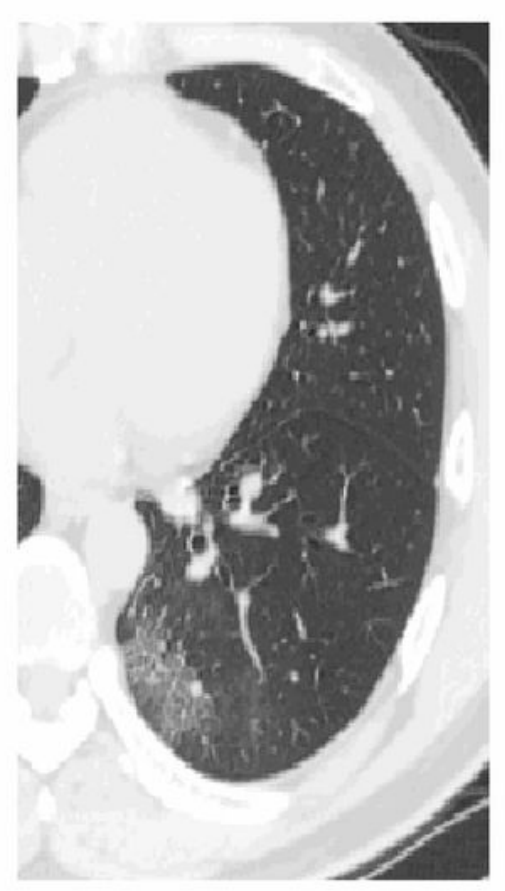

(A)

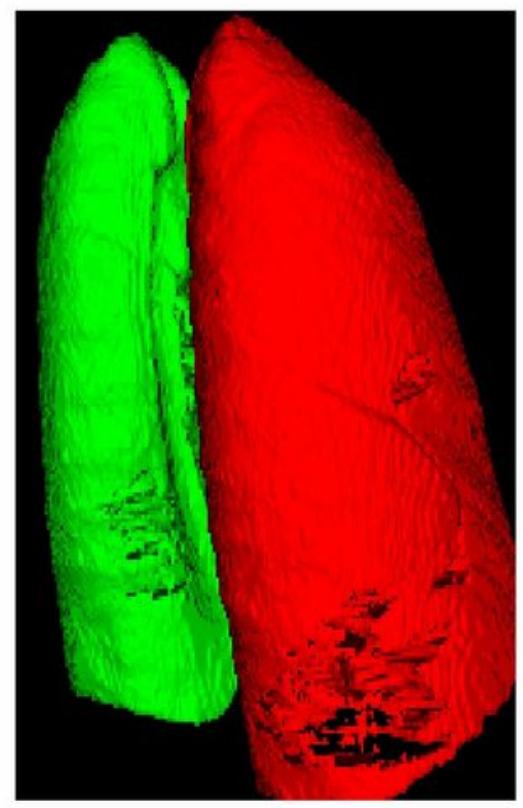

(D)

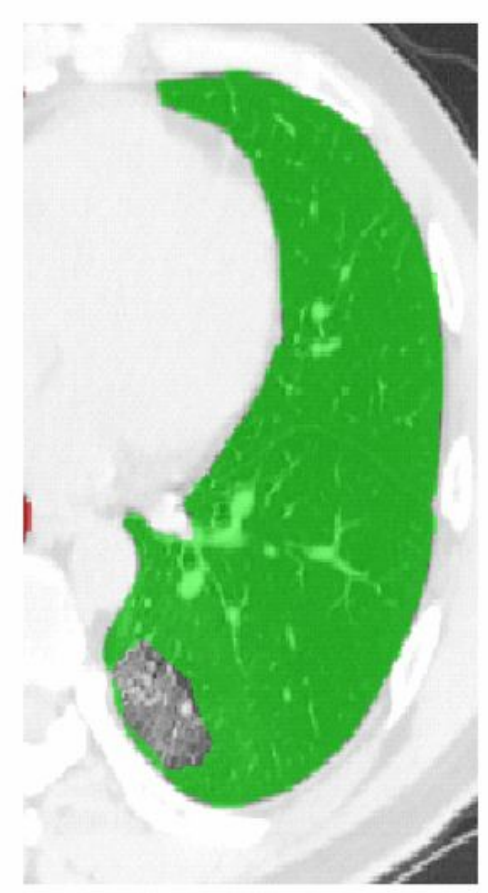

(B)

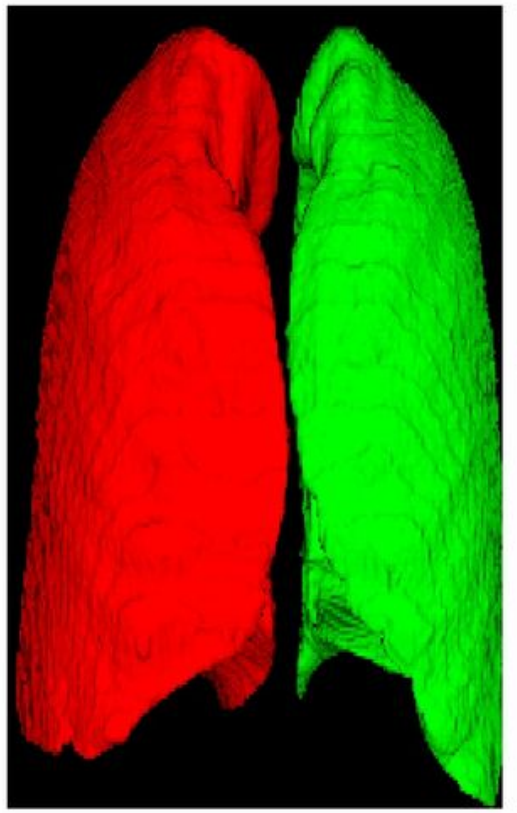

(E)

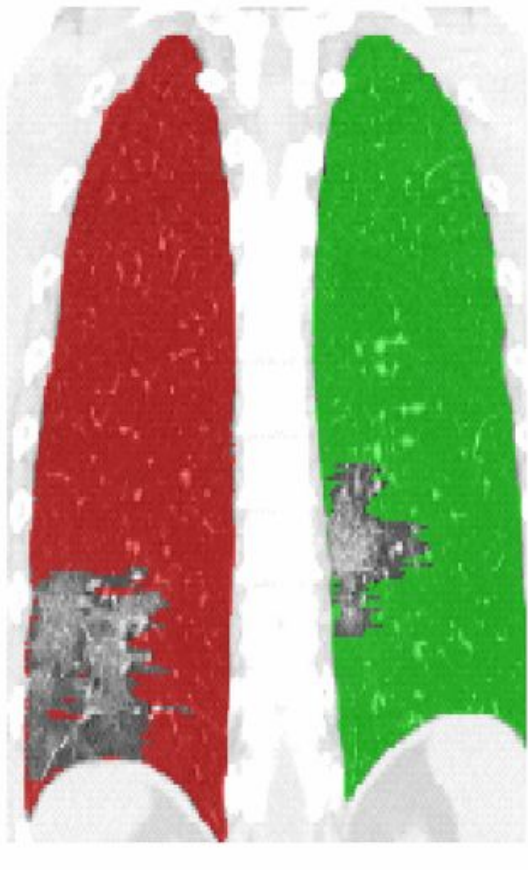

(C)

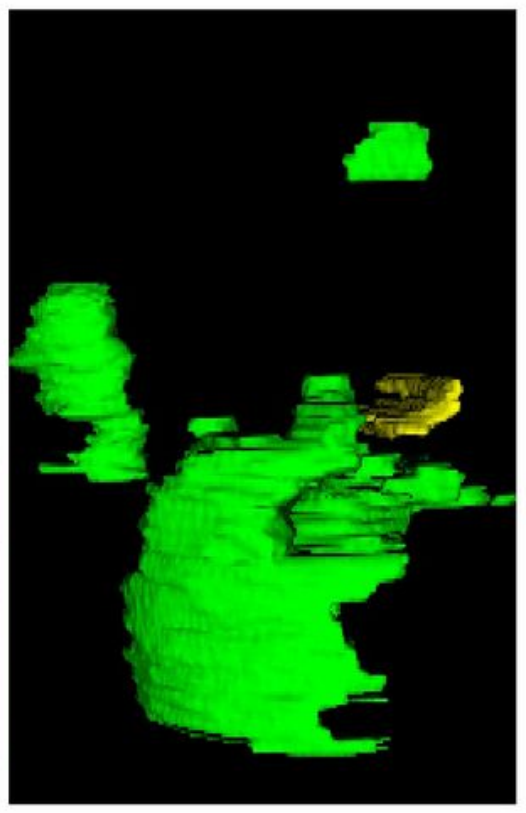

(F)

\section{Figure 2}

Illustration of imaging segmentation. A: axial view of CT image; B-C: delineation of lung abnormalities; DE: corrosion map, D indicated an involvement of dorsal lung, E showed none-involvement in the anterior part of lung; F: 3D regions of interests. 


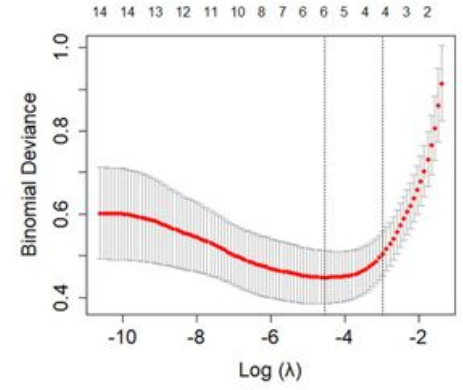

A

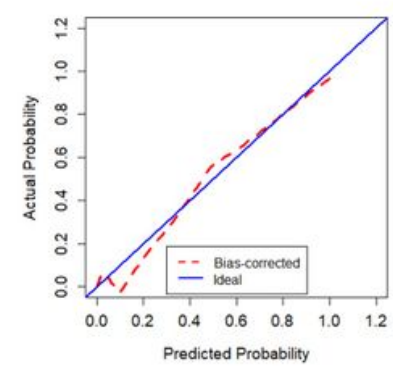

$\mathrm{D}$

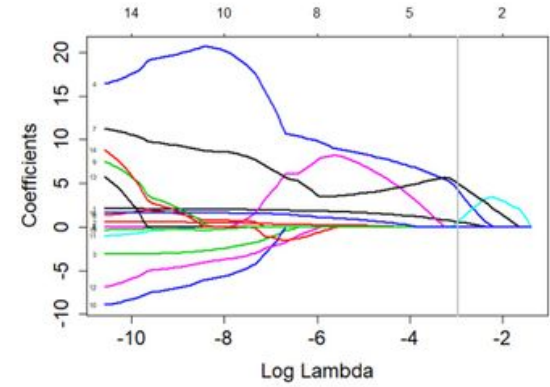

B

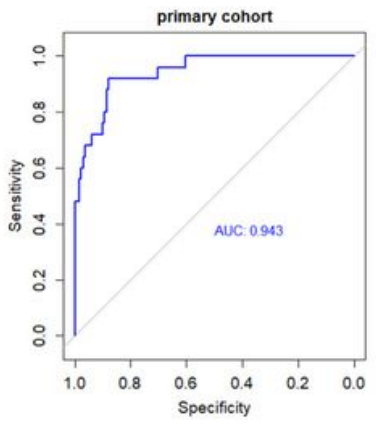

$\mathrm{E}$

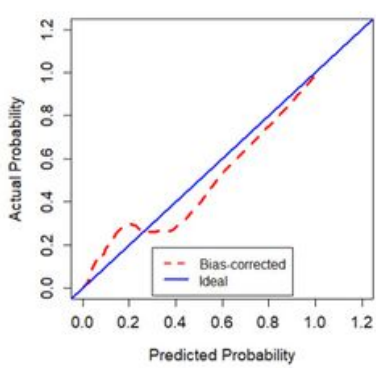

$\mathrm{C}$

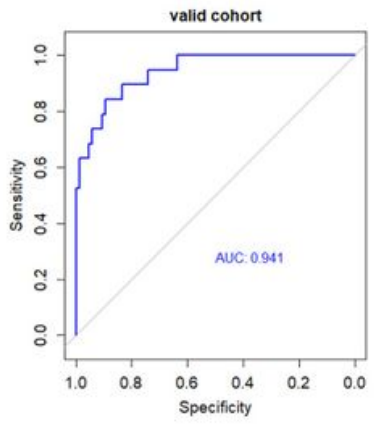

F

\section{Figure 3}

Feature selection and radiomics signature building using the least absolute shrinkage and selection operator (LASSO) binary logistic regression. A: The parameter $(\lambda)$ was screened by using 10 -fold crossvalidation method and parameter $(\lambda)$ between two dotted lines was the optimal values by using the minimum criteria and the 1 standard error of the minimum criteria (the 1-SE criteria). B: LASSO coefficient profiles of the 14 radiological features. A coefficient profile plot was conducted against the $\log (\lambda)$ sequence. $\lambda$ value of 0.052 , with $\log (\lambda)=-2.96$ was selected (1-SE criteria) based on 10 -fold crossvalidation. Vertical line was drawn at the value selected, which resulted in 4 non-zero coefficients. C-D: Significant difference in radiomics score was shown between none-severe and severe groups in primary (C) and validation cohorts (D). E-F: The receiver operating characteristic (ROC) curve for the radiomics signature. The calibration curves represented calibration of radiomics signature in terms of consistency between the predicted severe probabilities of COVID-19 and observed severe probabilities of COVID-19. The $x$-axis represents the predicted severe probabilities while the $y$-axis represents the actual probabilities. The actual probability was calculated by the formula PA $=[1+\exp -(a x+b)]-1$, where $x=\operatorname{logit}(p), p$ is the predicted probability, $a$ is the slope estimates, and $b$ is the corrected intercept. The $45^{\circ}$ dotted line represents the perfect prediction of an ideal model and the dotted lines represents the performance of the built nomogram model, a closer fit to the dotted line represents a better prediction. 


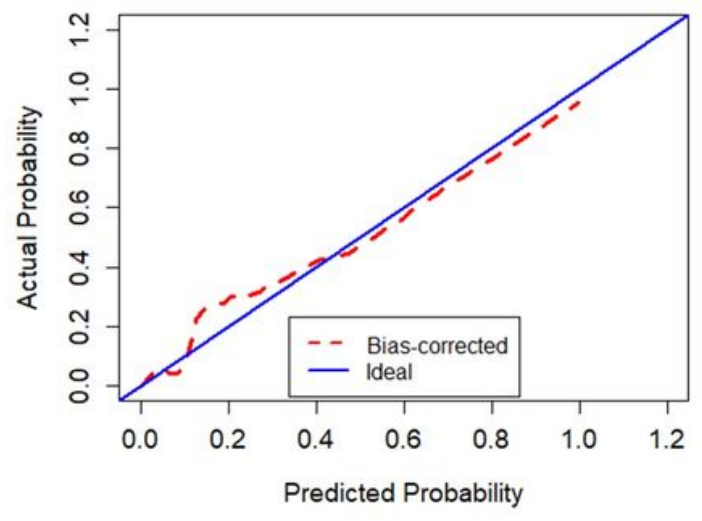

A

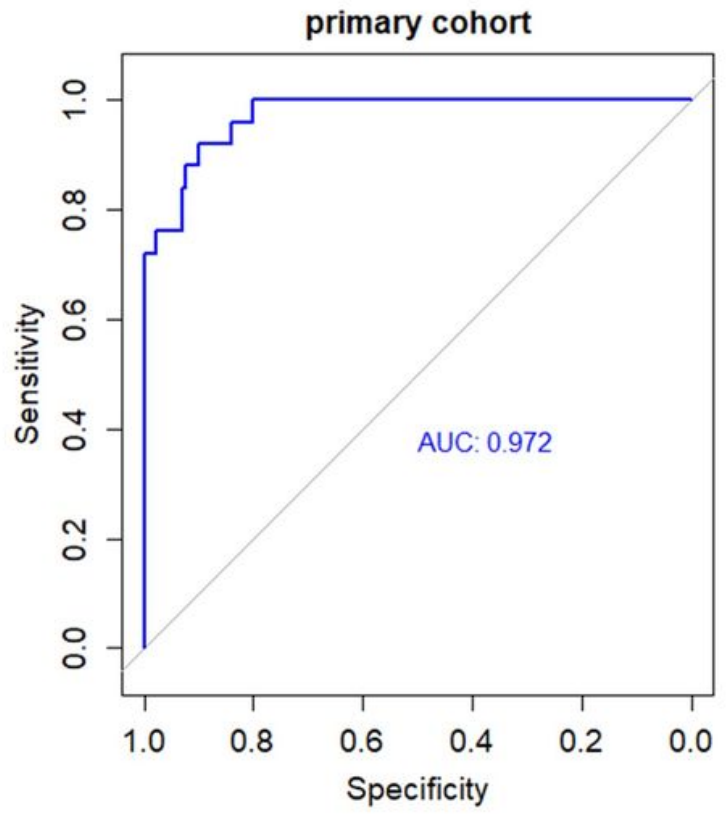

$\mathrm{C}$

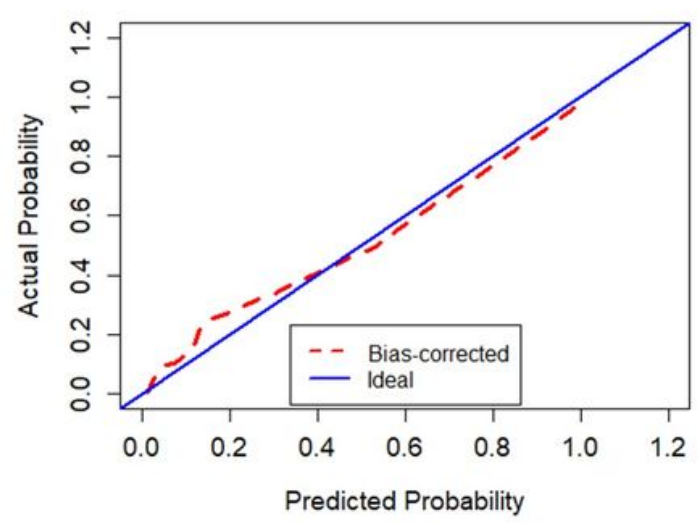

B

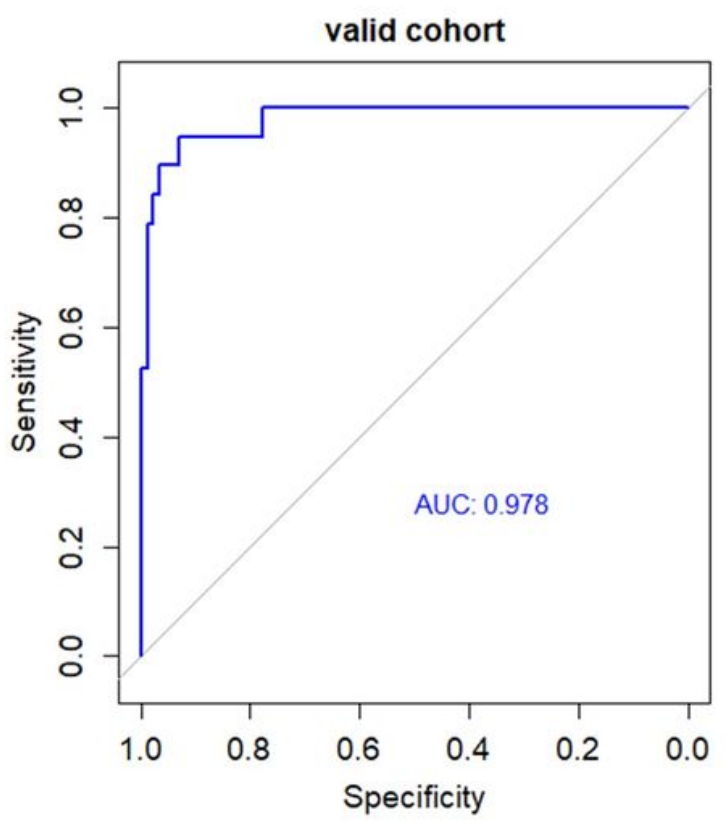

$\mathrm{D}$

\section{Figure 5}

Calibration curves and ROC curves of the radiomics nomogram. A-B: Calibration curves of the radiomics nomogram in the primary and validation cohorts, respectively. C-D: ROC curves of the radiomics nomogram in primary and validation cohorts, respectively. 


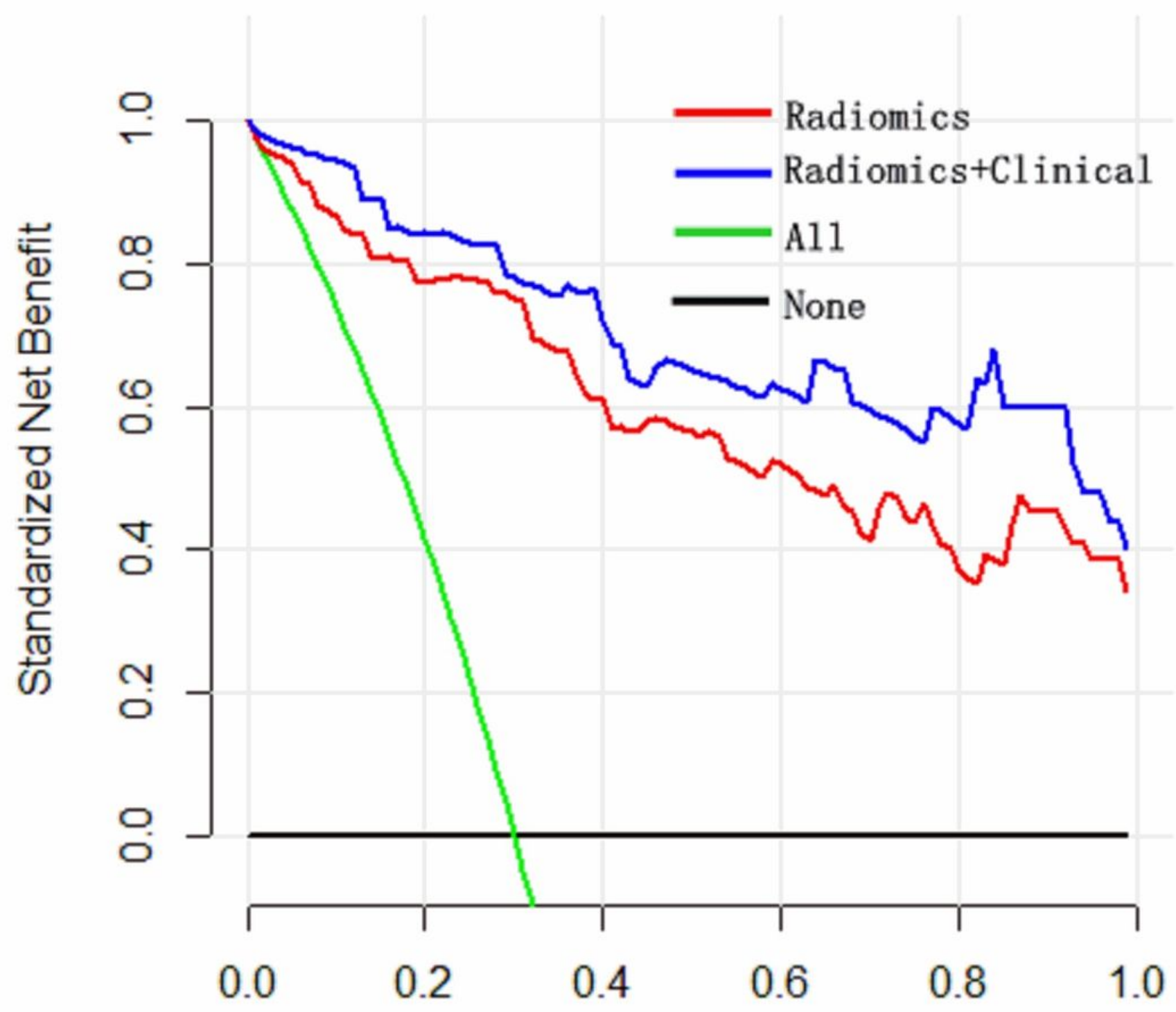

High Risk Threshold

Figure 6

Decision curve analysis for the radiomics nomogram. The $x$-axis represents the threshold probability and the $y$-axis measures the net benefit. The blue line represents the model combines the clinical features with radiomics scores and the pink line represents the radiomics nomogram. The green line assumes that all patients are severe of COVID-19. Thin black line hypothesizes that all patients are non-severe of COVID19. The calculation of net benefit was performed by subtracting the proportion of false positive from proportion of true positive in all patients, weighting with the relative harm of giving up treatment 
compared with the negative consequence of an unnecessary treatment[24]. The calculation of relative harm was performed by $(\mathrm{Pt} /(1-\mathrm{Pt}))$, $\mathrm{Pt}$ is the threshold probability, where the expected benefit of treatment is equal to the expected benefit of avoiding treatment. According to the decision curve, using the method which combines radiomics with clinic information to predict the probability of severe COVID-19 always adds more benefit than other three methods (only including radiomics, or the teat-all-patients scheme, or treat-none scheme). For example, if the personal threshold probability of a patient is $80 \%$ (the patient is willing to receive treatment if his probability of severe COVID-19 is $>80 \%$ ), then the benefit is about 0.6 when using the method that combines radiomics with clinic to decide whether to undergo treatment, which exhibits the best benefit compared with other three methods. 